\title{
A Stochastic Recurrence Equation Approach to Stationarity and phi-Mixing of a Class of Nonlinear ARCH Models
}

Francisco (F.) Blasques ${ }^{1}$

Marc Nientker ${ }^{2}$

1: VU Amsterdam, The Netherlands; Tinbergen Institute, The Netherlands

2: VU Amsterdam, The Netherlands 
Tinbergen Institute is the graduate school and research institute in economics of Erasmus University Rotterdam, the University of Amsterdam and VU University Amsterdam.

Contact: discussionpapers@tinbergen.nl

More TI discussion papers can be downloaded at the Tinbergen Site

Tinbergen Institute has two locations:

Tinbergen Institute Amsterdam

Gustav Mahlerplein 117

1082 MS Amsterdam

The Netherlands

Tel.: +31(0)20 5984580

Tinbergen Institute Rotterdam

Burg. Oudlaan 50

3062 PA Rotterdam

The Netherlands

Tel.: +31(0)10408 8900 


\title{
A Stochastic Recurrence Equation Approach to Stationarity and $\varphi$-Mixing of a Class of Nonlinear ARCH Models
}

\author{
Francisco Blasques $^{(a, b)}$ and Marc Nientker ${ }^{(a)}$ \\ (a) VU University Amsterdam \\ (b) Tinbergen Institute
}

August 2, 2017

\begin{abstract}
This article generalises the results of Saïdi and Zakoian (2006) to a considerably larger class of nonlinear ARCH models with discontinuities, leverage effects and robust news impact curves. We propose a new method of proof for the existence of a strictly stationary and $\varphi$-mixing solution. Moreover, we show that any path converges to this solution. The proof relies on stochastic recurrence equation theory and builds on the work of Bougerol (1993) and Straumann (2005). The assumptions that we need for this approach are less restrictive than those imposed in Saïdi and Zakoian (2006) and typically found in Markov chain theory, as they require very little from the distribution of the underlying process. Furthermore, they can be stated in a general setting for random functions on a separable Banach space as is done in Straumann and Mikosch (2006). Finally, we state sufficient conditions for the existence of moments.
\end{abstract}

\section{Introduction}

Since Engle (1982) introduced the autoregressive conditional heteroskedasticity (ARCH) model, many extensions and variations have been proposed in the literature to model the volatility of time series. These models typically define the dynamics of a heteroskedastic time series $\left(\epsilon_{t}\right)_{t \in \mathbb{Z}}$ as

$$
\begin{aligned}
\epsilon_{t} & =\sigma_{t} \eta_{t}, \\
\sigma_{t}^{2} & =\sigma\left(\epsilon_{t-1}, \epsilon_{t-2}, \ldots\right),
\end{aligned}
$$

where $\left(\eta_{t}\right)_{t \in \mathbb{Z}}$ is a strictly stationary and ergodic sequence of random variables and the volatility $\sigma_{t}$ is a positive and measurable function of the past $\left(\epsilon_{t-1}, \epsilon_{t-2}, \ldots\right)$. Important examples are the generalized ARCH (GARCH) model of Bollerslev (1986), the exponential GARCH model of Nelson (1991) and the threshold GARCH model introduced by Zakoian (1994).

In this paper we study a class of nonlinear ARCH processes that generalise the model introduced in Saïdi and Zakoian (2006), which takes the form

$$
\begin{aligned}
\epsilon_{t} & =\sigma_{t} \eta_{t}, \\
\sigma_{t}^{2} & =\omega+\alpha \epsilon_{t-1}^{2} \mathbb{1}\left\{\epsilon_{t-1}^{2}>k \epsilon_{t-2}^{2}\right\},
\end{aligned}
$$


where again $\left(\eta_{t}\right)_{t \in \mathbb{Z}}$ is a strictly stationary and ergodic sequence of random variables, the parameters $\alpha$ and $k$ are nonnegative and $\omega$ is positive. Note that (1.1) includes the traditional $\operatorname{ARCH}(1)$ at $k=0$ and the conditional homoskedastic model, a model with constant volatility, at $\alpha=0$. In fact, model (1.1) is an endogenous regime switching model between these two specifications. The model changes from the constant volatility regime to the $\mathrm{ARCH}(1)$ specification when the relative variation $\epsilon_{t-1}^{2} / \epsilon_{t-2}^{2}$ becomes large, indicating a setting in which it is more likely for the volatility to be time varying. See the original paper for a more extensive discussion of the model and Saïdi (2003) for empirical motivation based on financial time series data.

The stability properties of (1.1) are non standard because the updating equation has a discontinuity. Firstly, this creates nonlinear dynamics so that the theory on Lyapunov exponents as developed in Bougerol and Picard (1992a,b) cannot be used. Secondly, stochastic recurrence equation theory as developed in Bougerol (1993) and Straumann (2005) cannot be directly applied. That approach in general tries to expand the model back into the infinite past and attempts to show that this converges to a limit. This limit can then be shown to be a solution of the model with nice properties such as stationarity. However, nonlinear models often make expanding backwards next to impossible and thus one has to resort to imposing contraction conditions involving Lipschitz continuity to ensure convergence. The discontinuity in our updating equation violates those assumptions.

Saïdi and Zakoian (2006) avoid the described problems by opting for another approach based on Markov chain theory to study the stability properties of (1.1). They show the existence of a stationary and $\beta$-mixing solution, under the condition that the distribution of the underlying process $\left(\eta_{t}\right)_{t \in \mathbb{Z}}$ is independent and identically distributed (iid), has strict positive density and fixed moments $\mathbb{E}\left(\eta_{t}\right)=0$ and $\mathbb{E}\left(\eta_{t}^{2}\right)=1$. This paper extends the stochastic recurrence equation theory by introducing new assumptions that allow for the discontinuity and ensure backward expanding converges. These assumptions are based on the observation that there is a positive probability for the volatility to be constant, that is $\sigma_{t}^{2}$ equals $\omega$, irrespective of the past volatility $\left(\sigma_{s}^{2}\right)_{s<t}$. Under moderate conditions, this will occur for infinitely many $t \in \mathbb{Z}$ and thus expanding backwards into the past becomes a finite operation, which immediately guarantees convergence. With our approach we show the existence of a stationary and ergodic or $\varphi$-mixing solution, and that any path converges to this solution at any rate. The assumptions needed to get these results are less strict than those imposed in Saïdi and Zakoian (2006). Moreover, the condition that $\sigma_{t}^{2}$ equals $\omega$ with positive probability can easily be extended to a general setting for random functions on a separable Banach space: the functions need to have a positive probability to be constant. This allows us to model the time varying variables $\epsilon_{t}$ and $\sigma_{t}$ as functions of the model parameters, which can be used to obtain stronger inference results as is done in Straumann and Mikosch (2006). Moreover, the theory extends to a general setting beyond volatility models.

In what follows, Section 2 discusses stability conditions for random functions on separable Banach spaces and states our main results in their most general form. Section 3 specifies a class of nonlinear ARCH models that contains model (1.1), applies the results of Section 2 and discusses some examples including leverage effects and robust news impact curves. Section 4 derives a sufficient condition for the existence of moments to the class of models in Section 3 and applies it to the examples introduced. 


\section{General results}

The aim of this section is to prove our main results on the existence of a stationary ergodic solution to which all paths converge (Theorem 2.1) and the existence of a solution that is $\varphi$-mixing at geometric rate (Theorem 2.4). We will base our treatment of stability on stochastic recurrence equations (SREs) as is done in Straumann (2005) and Straumann and Mikosch (2006). The main advantage of stochastic recurrence equation (SRE) techniques is that they are very general. For example, proposition 7.6 in Kallenberg (2002) proves that any homogeneous Markov chain can be seen as a solution to a SRE. We refer the reader to Diaconis and Freedman (1999) for a thorough overview of SREs.

Let $S$ be a closed subset of a separable Banach space equipped with a norm $\|\cdot\|$ and Borel sigma-algebra $\mathcal{B}(S)$ and let $(E, \mathcal{E})$ be a measurable space. Let $\left(\eta_{t}\right)_{t \in \mathbb{Z}}$ be a sequence of stochastic elements taking values in $E$ and let $\phi: S \times E \rightarrow S$ be a measurable map. Then we can define a sequence of random functions $\left(\phi_{t}\right)_{t \in \mathbb{Z}}$ by setting $\phi_{t}:=\phi\left(\cdot, \eta_{t}\right)$. Let $T$ be either $\mathbb{Z}$ or $\mathbb{N}$. A stochastic process $\left(X_{t}\right)_{t \in T}$ taking values in $S$ that satisfies

$$
X_{t+1}=\phi_{t}\left(X_{t}\right) \quad \forall t \in T
$$

is said to be a solution to the SRE associated with $\left(\phi_{t}\right)_{t \in \mathbb{Z}}$ if $T=\mathbb{Z}$, and a partial solution if $T=\mathbb{N}$. We now construct a specific possible solution $\left(Y_{t}\right)_{t \in \mathbb{Z}}$ to $(2.1)$ by using the backward iterates defined as $\phi_{t}^{(0)}=\operatorname{Id}_{S}$ and

$$
\phi_{t}^{(m)}=\phi_{t} \circ \phi_{t-1} \cdots \circ \phi_{t-m+1}, \quad m \in \mathbb{N} .
$$

Let $x \in S$ be an element such that

$$
Y_{t+1}:=\lim _{m \rightarrow \infty} \phi_{t}^{(m)}(x)
$$

exists almost surely for all $t \in \mathbb{Z}$. Bougerol (1993) and Straumann and Mikosch (2006) show that this is the case under appropriate regularity conditions involving the contracting behavior of each $\phi_{t}$ and the distribution of $\left(\phi_{t}\right)_{t \in \mathbb{Z}}$. Moreover, they show that the sequence of limits $\left(Y_{t}\right)_{t \in \mathbb{Z}}$ is then the unique ergodic solution to (2.1) and that any partial solution converges to this unique one at a geometric rate as $t \rightarrow \infty$. In this article we pursue a similar approach, we also focus on the limit of the backward iterates in (2.2), show that it is well defined and that the resulting sequence $\left(Y_{t}\right)_{t \in \mathbb{Z}}$ possesses the right properties. However, we rely on considerably different conditions. In particular, we do not make use of the contraction condition in Bougerol (1993).

Assumption A. The sequence $\left(\phi_{t}\right)_{t \in \mathbb{Z}}$ satisfies the following conditions:

A1. The function $\phi$ is $\mathcal{B}(S) \times \mathcal{E} / \mathcal{B}(S)$ measurable.

A2. The sequence $\left(\eta_{t}\right)_{t \in \mathbb{Z}}$ is strictly stationary ergodic.

A3. There exists an $M \in \mathbb{N}$, an event $A \in \mathcal{E}^{M}$ and a $c \in S$ such that $\left(\eta_{t}, \eta_{t-1}, \ldots, \eta_{t-M+1}\right) \in$ $A$ with positive probability and

$$
\left(\eta_{t}, \eta_{t-1}, \ldots, \eta_{t-M+1}\right) \in A \quad \Rightarrow \quad \phi_{t}^{(M)}(x)=c \quad \forall x \in S
$$


Condition A1 is rather weak and designed to ensure that backward iterates of $\left(\phi_{t}\right)_{t \in \mathbb{Z}}$ evaluated at any point $x \in S$ are proper random variables in $S$. Condition A2 is common in the literature on SREs, note that it is less strict than assuming that the sequence $\left(\eta_{t}\right)_{t \in \mathbb{Z}}$ is independent and identically distributed. An in depth discussion on stationarity and ergodicity can, for example, be found in chapter one of Krengel (1985). Condition A3 is less common, but generalises the main feature of the nonlinear ARCH model studied in Saïdi and Zakoian (2006). Specifically, condition A3 defines a model where the random function $\phi_{t}$ has a positive probability of being equal to a constant function with value $c$.

Under Assumption A we can prove that the limit of the backward iterates (2.2) exists, by showing that the sequence of backward iterates $\left(\phi_{t}^{(m)}(x)\right)_{m \in \mathbb{N}}$ is almost surely eventually constant. The proof relies on the fact that events of positive probability occur infinitely often over time in a strictly stationary ergodic sequence. Therefore the event $\left(\eta_{t}, \eta_{t-1}, \ldots, \eta_{t-M+1}\right) \in A$ occurs for infinitely many $t \in \mathbb{Z}$ and thus the limit of the backward iterates at such a $t$ then trivially exists and is equal to $c$. Uniqueness and convergence of paths follow from the same observation, since any two paths in model (2.1) will coincide at all such $t$, and therefore must be the same (eventually).

Theorem 2.1. Let Assumption $A$ hold and $x \in S$. Then the sequence $\left(\phi_{t}^{(m)}(x)\right)_{m \in \mathbb{N}}$ is almost surely eventually constant for all $t \in \mathbb{Z}$. Consequently, $\left(Y_{t}\right)_{t \in \mathbb{Z}}$ is well defined, strictly stationary ergodic and the unique solution to (2.1). Moreover, for any partial solution $\left(\tilde{Y}_{t}\right)_{t \in \mathbb{N}}$ and function $f: \mathbb{N} \rightarrow \mathbb{R}$ we have $\lim _{t \rightarrow \infty} f(t)\left\|Y_{t}-\tilde{Y}_{t}\right\|=0$.

We have to discuss some preliminary results on strictly stationary ergodic (SE) sequences before we can prove Theorem 2.1. One reason that SE sequences play a big role in time series analysis is that they satisfy the conditions needed for Birkhoff's ergodic theorem, Birkhoff (1931). This theorem applied to an SE sequence of real valued random variables $\left(X_{t}\right)_{t \in \mathbb{N}}$ states that if $\mathbb{E}\left|X_{1}\right|<\infty$, then almost surely

$$
\lim _{n \rightarrow \infty} \frac{1}{n} \sum_{t=1}^{n} X_{t}=E\left(X_{1}\right) .
$$

SE sequences are also easy to manipulate to create new SE sequences. We provide two results from Straumann (2005).

Lemma 2.2. Let $(E, \mathcal{E})$ and $(\tilde{E}, \tilde{\mathcal{E}})$ be two measurable spaces, let $\left(X_{t}\right)_{t \in \mathbb{Z}}$ be an SE sequence of $E$-valued random elements and let $f: E^{\mathbb{N}} \rightarrow \tilde{E}$ be a $\mathcal{E}^{\mathbb{N}} / \tilde{\mathcal{E}}$ measurable function. Then the sequence of $\tilde{E}$-valued random elements $\left(\tilde{X}_{t}\right)_{t \in \mathbb{Z}}$ defined as $\tilde{X}_{t}=f\left(X_{t}, X_{t-1}, \ldots\right)$ is $S E$.

Proof. See proposition 2.1.1 in Straumann (2005).

Lemma 2.3. Let $(E, \mathcal{E})$ be a measurable space and let $(S, \mathcal{B}(S))$ be a closed subset of a separable Banach space endowed with its Borel sigma-algebra. Let $\left(X_{t}\right)_{t \in \mathbb{Z}}$ be a SE sequence of E-valued random elements and let $\left(f_{m}\right)_{m \in \mathbb{N}}$ be a sequence of functions $E^{\mathbb{N}} \rightarrow S$ that are $\mathcal{E}^{\mathbb{N}} / \mathcal{B}(S)$ measurable. Suppose that there exists a $t \in \mathbb{Z}$ such that

$$
\lim _{m \rightarrow \infty} f_{m}\left(X_{t}, X_{t-1}, \ldots\right)
$$

exists almost surely. Then there exists a function $f: E^{\mathbb{N}} \rightarrow S$ that is $\mathcal{E}^{\mathbb{N}} / \mathcal{B}(S)$ measurable and satisfies

$$
\tilde{X}_{t}:=\lim _{m \rightarrow \infty} f_{m}\left(X_{t}, X_{t-1}, \ldots\right)=f\left(X_{t}, X_{t-1}, \ldots\right)
$$


for all $t \in \mathbb{Z}$. Moreover, the sequence of $S$-valued random elements $\left(\tilde{X}_{t}\right)_{t \in \mathbb{Z}}$ is $S E$.

Proof. See corollary 2.1.3 in Straumann (2005).

Proof of Theorem 2.1. Fix a $t \in \mathbb{Z}$. We begin by proving that $\left(\phi_{t}^{(m)}(x)\right)_{m \in \mathbb{N}}$ is almost surely eventually constant. Define for $s \geq 0$,

$$
I_{s}=\mathbb{1}\left\{\left(\eta_{t-s}, \eta_{t-s-1}, \ldots, \eta_{t-s-M+1}\right) \in A\right\} .
$$

The sequence $\left(I_{s}\right)_{s \geq 0}$ is SE by Lemma 2.2. Then, by Birkhoff's ergodic theorem, almost surely

$$
\lim _{n \rightarrow \infty} \frac{1}{n} \sum_{s=0}^{n-1} I_{s}=E\left(I_{0}\right)=\mathbb{P}\left(\left(\eta_{t}, \eta_{t-1}, \ldots, \eta_{t-M+1}\right) \in A\right)>0 .
$$

This implies that the event $I_{s}=1$ occurs almost surely for infinitely many $s \geq 0$. Therefore we can choose the smallest such $s$, note that it is a random variable, and conclude that

$$
\phi_{t}^{(m)}(x)=\phi_{t}^{(s)}\left(\phi_{t-s}^{(m-s)}(x)\right)=\phi_{t}^{(s)}\left(\phi_{t-s}^{(M)}\left(\phi_{t-s-M}^{(m-s-M)}(x)\right)\right)=\phi_{t}^{(s)}(c)
$$

for all $m \geq s+M$. It follows by Lemma 2.3 that the sequence $\left(Y_{t}\right)_{t \in \mathbb{Z}}$ is well defined and SE. Moreover, for $s=0$ we get $Y_{t+1}=c=\phi_{t}\left(Y_{t}\right)$, while for $s \geq 1$ we have

$$
Y_{t+1}=\lim _{m \rightarrow \infty} \phi_{t}^{(m)}(x)=\phi_{t}^{(s)}(c)=\phi_{t}\left(\phi_{t-1}^{(s-1)}(c)\right)=\phi_{t}\left(\lim _{m \rightarrow \infty} \phi_{t-1}^{(m)}(x)\right)=\phi_{t}\left(Y_{t}\right) .
$$

Therefore $\left(Y_{t}\right)_{t \in \mathbb{Z}}$ is a solution to $(2.1)$. If $\left(X_{t}\right)_{t \in \mathbb{Z}}$ is any other solution to $(2.1)$, then

$$
X_{t+1}=\phi_{t}^{(s)}\left(\phi_{t-s}^{(M)}\left(X_{t-s-M}\right)\right)=\phi_{t}^{(s)}(c)=Y_{t+1}
$$

and hence it is identical to $\left(Y_{t}\right)_{t \in \mathbb{Z}}$.

It remains to prove the final statement. Similarly as before, we can almost surely find an $s>M-1$ such that $\left(\eta_{s}, \eta_{s-1}, \ldots, \eta_{s-M+1}\right) \in A$ and thus

$$
Y_{t+1}=\phi_{t}^{(t-s)}\left(\phi_{s}^{(M)}\left(Y_{s-M+1}\right)\right)=\phi_{t}^{(t-s)}(c)=\phi_{t}^{(t-s)}\left(\phi_{s}^{(M)}\left(\tilde{Y}_{s-M+1}\right)\right)=\tilde{Y}_{t+1}
$$

for all $t \geq s$. We conclude that

$$
\lim _{t \rightarrow \infty} f(t)\left\|Y_{t}-\tilde{Y}_{t}\right\|=0
$$

irrespective of the function $f$, because $\left\|Y_{t}-\tilde{Y}_{t}\right\|$ is almost surely eventually zero.

A consequence of Theorem 2.1 is that we can derive sufficient conditions for the process $\left(Y_{t}\right)_{t \in \mathbb{Z}}$ to be $\varphi$-mixing. Let $\left(X_{t}\right)_{t \in \mathbb{Z}}$ be a stationary process and let $\mathcal{F}_{s}^{t}$, for $-\infty \leq s<$ $t \leq \infty$, denote the sigma algebra generated by $\left(X_{s}, X_{s+1}, \ldots, X_{t}\right)$. Then the $\varphi$-mixing coefficients for $\left(X_{t}\right)_{t \in \mathbb{Z}}$ are given by

$$
\varphi_{X}(t)=\sup _{C \in \mathcal{F}_{-\infty}^{0}, D \in \mathcal{F}_{t}^{\infty}, \mathbb{P}(C)>0}|\mathbb{P}(D \mid C)-\mathbb{P}(D)|
$$

and the process is called $\varphi$-mixing if $\varphi_{X}(t) \rightarrow 0$ as $t \rightarrow \infty$. 
Theorem 2.4. Suppose Assumption $A$ holds and that additionally $\left(\eta_{t}\right)_{t \in \mathbb{Z}}$ is $\varphi$-mixing with geometric rate. Then $\left(Y_{t}, \eta_{t}\right)_{t \in \mathbb{Z}}$ is $\varphi$-mixing with geometric rate.

The proof will depend on Theorem 2.1 as follows. Usually $Y_{t+1}$ depends on the entire past $\left(\eta_{t}, \eta_{t-1}, \ldots\right)$. However, if the event $\left(\eta_{t-s}, \eta_{t-s-1}, \ldots, \eta_{t-s-M+1}\right) \in A$ occurs for some $s \geq 0$, then $Y_{t+1}=\phi_{t}^{(s)}(c)$ and thus $Y_{t+1}$ depends only on $\left(\eta_{t}, \ldots, \eta_{t-s+1}\right)$. Therefore it will be enough to show that the probability that $s$ is large vanishes at a geometric rate. To show this we need the following two lemma's.

Lemma 2.5. Let $(E, \mathcal{E})$ and $(\tilde{E}, \tilde{\mathcal{E}})$ be two measurable spaces, let $\left(X_{t}\right)_{t \in \mathbb{Z}}$ be a sequence of $E$-valued random elements that is $\varphi$-mixing (with geometric rate). For a $m \in \mathbb{N}$ we denote $f: E^{m} \rightarrow \tilde{E}$ to be a $\mathcal{E}^{m} / \tilde{\mathcal{E}}$ measurable function. Then the sequence of $\tilde{E}$-valued random elements $\left(\tilde{X}_{t}\right)_{t \in \mathbb{Z}}$ defined as

$$
\tilde{X}_{t}=f\left(X_{t}, \ldots, X_{t-m}\right)
$$

is $\varphi$-mixing (with geometric rate).

Proof. The sigma-algebra generated by $\left(\ldots, \tilde{X}_{-1}, \tilde{X}_{0}\right)$ is contained in the sigma-algebra generated by $\left(\ldots, X_{-1}, X_{0}\right)$. Similarly, the sigma-algebra generated by $\left(\tilde{X}_{t}, \tilde{X}_{t+1}, \ldots\right)$ is contained in the sigma-algebra generated by $\left(X_{t-m}, X_{t-m+1}, \ldots\right)$. Therefore $\varphi_{\tilde{X}}(t) \leq$ $\varphi_{X}(t-m)$ for all $t \geq m$.

Lemma 2.6. Let $(E, \mathcal{E})$ be a measurable space and let $\left(X_{i}\right)_{i \in \mathbb{Z}}$ be a strictly stationary sequence of E-valued random elements that is $\varphi$-mixing. Then for any $B \in \mathcal{E}$ such that $\mathbb{P}\left(X_{1} \notin B\right)<1$, we have

$$
\mathbb{P}\left(\bigcap_{i=1}^{t}\left\{X_{i} \notin B\right\}\right) \rightarrow 0 \quad \text { as } \quad t \rightarrow \infty
$$

at a geometric rate.

Proof. For a real number $z \in \mathbb{R}$ we write $\lfloor z\rfloor$ to denote the largest integer that is not larger than $z$. Also, we use the ; symbol to denote joint probabilities. For any integer $k \leq t$ we have

$$
\begin{aligned}
& \mathbb{P}\left(X_{t} \notin B ; \ldots ; X_{1} \notin B\right) \\
& \quad=\prod_{i=0}^{\lfloor t / k\rfloor-1} \mathbb{P}\left(X_{t-i k} \notin B ; \ldots ; X_{t-(i+1) k+1} \notin B \mid X_{t-(i+1) k} \notin B ; \ldots ; X_{1} \notin B\right) \\
& \leq \prod_{i=0}^{\lfloor t / k\rfloor-1} \mathbb{P}\left(X_{t-i k} \notin B \mid X_{t-(i+1) k} \notin B ; \ldots ; X_{1} \notin B\right) \\
& \leq \prod_{i=0}^{\lfloor t / k\rfloor-1} \mathbb{P}\left(X_{t-i k} \notin B\right)+\varphi_{X}(k) \\
&=\left(\mathbb{P}\left(X_{1} \notin B\right)+\varphi_{X}(k)\right)^{\lfloor t / k\rfloor-1} .
\end{aligned}
$$

Choose $k$ big enough such that $\mathbb{P}\left(X_{1} \notin B\right)+\varphi_{X}(k)<1$, which can be done since $\varphi_{X}(k) \rightarrow 0$ as $k \rightarrow \infty$. Note that if any of the events that we conditioned on has probability zero, 
then the lemma follows immediately.

Proof of Theorem 2.4. For $-\infty \leq s<t \leq \infty$ we write $\mathcal{G}_{s}^{t}$ to denote the sigma algebra generated by $\left(\eta_{s}, \eta_{s+1}, \ldots, \eta_{t}\right)$ and $\mathcal{H}_{s}^{t}$ to denote the sigma algebra generated by $\left(\left(Y_{s}, \eta_{s}\right),\left(Y_{s+1}, \eta_{s+1}\right), \ldots,\left(Y_{t}, \eta_{t}\right)\right)$. Fix $t \in \mathbb{N}$ and let $s \geq 0$ again be the random variable that denotes the smallest number such that $\left(\eta_{t-s}, \eta_{t-s-1}, \ldots, \eta_{t-s-M+1}\right) \in A$ occurs. Then we have $Y_{t+1}=\lim _{m \rightarrow \infty} \phi_{t}^{(m)}(x)=\phi_{t}^{(s)}(c)$. Therefore, for a $B \in \mathcal{B}(S)$ and a $k \geq 0$ the event $\left\{Y_{t+1} \in B ; s \leq k\right\} \in \mathcal{G}_{t-k-M+1}^{t}$, since $\{s \leq k\} \in \mathcal{G}_{t-k-M+1}^{t}$. Similarly, for any $D \in \mathcal{H}_{t}^{\infty}$ the event $D \cap\{s \leq t / 2-M+1\} \in \mathcal{G}_{\lceil t / 2\rceil}^{\infty}$, where we write $\lceil z\rceil$ to denote the smallest integer that is not smaller than $z$. It follows for $C \in \mathcal{H}_{-\infty}^{0} \subseteq \mathcal{G}_{-\infty}^{0}$, by partitioning on $s \leq t / 2-M+1$ and its complement, that

$$
|\mathbb{P}(D \mid C)-\mathbb{P}(D)| \leq \varphi_{\eta}(\lceil t / 2\rceil)+\mathbb{P}(D ; s>t / 2-M+1 \mid C)+\mathbb{P}(D ; s>t / 2-M+1) .
$$

Since $\{s>t / 2-M+1\} \in \mathcal{G}_{\lceil t / 2\rceil}^{t}$ we get

$$
\mathbb{P}(D ; s>t / 2-M+1 \mid C) \leq \mathbb{P}(s>t / 2-M+1 \mid C) \leq \mathbb{P}(s>t / 2-M+1)+\varphi_{\eta}(\lceil t / 2\rceil) .
$$

It follows that

$$
\varphi_{(Y, \eta)}(t) \leq 2 \varphi_{\eta}(\lceil t / 2\rceil)+2 \mathbb{P}(s>t / 2-M+1) .
$$

The first term goes geometrically fast to zero by assumption. For the second part we define $X_{t}=\left(\eta_{t}, \eta_{t-1}, \ldots, \eta_{t-M+1}\right)$. Then $\left(X_{t}\right)_{t \in \mathbb{Z}}$ is $\varphi$-mixing by Lemma 2.5. Therefore, by Lemma 2.6 , and the fact that $\mathbb{P}\left(X_{t} \in A\right)>0$, we have

$$
\mathbb{P}(s>t / 2-M+1)=\mathbb{P}\left(\bigcap_{i=0}^{\lceil t / 2\rceil}\left\{X_{t-i} \notin A\right\}\right) \rightarrow 0
$$

geometrically fast as $t \rightarrow \infty$.

\section{Application to Nonlinear ARCH}

We now introduce a general nonlinear ARCH model that contains the model of Saïdi and Zakoian (2006) and illustrate how to apply our main results of Section 2. Let $u: \mathbb{R}^{2} \rightarrow$ $[0, \infty)$ be a nonnegative Borel measurable function that possibly depends on a vector of parameters $\theta$ that lie in a parameter space $\Theta$. The general model of interest is given by

$$
\begin{aligned}
\epsilon_{t} & =\sigma_{t} \eta_{t}, \\
\sigma_{t}^{2} & =\omega+u\left(\epsilon_{t-1}, \sigma_{t-1}^{2} ; \theta\right) \mathbb{1}\left\{\epsilon_{t-1}^{2}>k \epsilon_{t-2}^{2}\right\},
\end{aligned}
$$

where $\omega$ and $k$ are strictly positive. The generalisation compared to (1.1) is that we replace the term $\alpha \epsilon_{t-1}^{2}$ with a general updating function $u$. We discuss model (1.1) and other examples in Section 3.1.

We start by analysing the dynamics concerning the time varying volatility. Given that $u$ is nonnegative we immediately see that any possible solution to (3.1) must satisfy $\sigma_{t}^{2} \in I:=[\omega, \infty)$. Assuming that the model is well specified, we get

$$
\sigma_{t}^{2}=\omega+\tilde{u}\left(\sigma_{t-1}^{2}, \eta_{t-1} ; \theta\right) \mathbb{1}\left\{\sigma_{t-1}^{2} \eta_{t-1}^{2}>k \sigma_{t-2}^{2} \eta_{t-2}^{2}\right\},
$$


where $\tilde{u}\left(\sigma_{t-1}^{2}, \eta_{t-1} ; \theta\right)=u\left(\epsilon_{t-1}\left(\sigma_{t-1}, \eta_{t-1}\right), \sigma_{t-1}^{2} ; \theta\right)$. Our analysis will focus on this model, since any solution to (3.2) can be used to create a solution to (3.1). Note that $\sigma_{t}^{2}$ depends both on $\sigma_{t-1}^{2}$ and $\sigma_{t-2}^{2}$. The random functions $\left(\phi_{t}\right)_{t \in \mathbb{Z}}$ associated with (3.2) will therefore be defined on $I^{2}$ and are given by

$$
\phi_{t-1}(x, y)=\phi\left(x, y, \eta_{t-1}, \eta_{t-2}\right)=\omega+\tilde{u}\left(x, \eta_{t-1} ; \theta\right) \mathbb{1}\left\{x \eta_{t-1}^{2}>k y \eta_{t-2}^{2}\right\} .
$$

Unfortunately these are not in the framework of the SRE theory in Section 2, since $\phi$ : $I^{2} \times \mathbb{R}^{2} \rightarrow I$. Therefore we will look at the two dimensional model

$$
\left(\sigma_{t}^{2}, \sigma_{t-1}^{2}\right)=\left(\phi_{t-1}\left(\sigma_{t-1}^{2}, \sigma_{t-2}^{2}\right), \sigma_{t-1}^{2}\right)
$$

which has state space $S:=I^{2}$. The random functions associated with (3.3) are given by

$$
\psi_{t-1}(x, y)=\psi\left(x, y, \eta_{t-1}, \eta_{t-2}\right)=\left(\phi_{t-1}(x, y), x\right)
$$

Define $\phi_{t}^{(-1)}(x, y)=y$ and $\phi_{t}^{(0)}(x)=x$, then the backward iterates for $m \in \mathbb{N}$ are given by

$$
\begin{aligned}
& \phi_{t}^{(m)}(x, y)=\phi_{t}\left(\phi_{t-1}^{(m-1)}(x, y), \phi_{t-2}^{(m-2)}(x, y)\right), \\
& \psi_{t}^{(m)}(x, y)=\left(\phi_{t}^{(m)}(x, y), \phi_{t-1}^{(m-1)}(x, y)\right) .
\end{aligned}
$$

We now state the weakest assumption for our nonlinear ARCH model that ensures we satisfy Assumption A and therefore obtain the results from Theorems 2.1 and 2.4. This result is derived in Theorem 3.2.

\section{Assumption B.}

B1. The sequence $\left(\eta_{t}\right)_{t \in \mathbb{Z}}$ is SE.

B2. The following event has positive probability of occurring:

$$
\eta_{t}^{2} \leq \inf _{x \in I} \frac{k x \eta_{t-1}^{2}}{\omega+\tilde{u}\left(x, \eta_{t-1}^{2} ; \theta\right)} \quad \text { and } \quad \eta_{t-1}^{2} \leq \inf _{x \in I} \frac{k x \eta_{t-2}^{2}}{\omega+\tilde{u}\left(x, \eta_{t-2}^{2} ; \theta\right)} .
$$

Assumption B is very general, but quite complex and thus hard to interpret. It is a restriction on the joint probability law of $\left(\eta_{t}, \eta_{t-1}, \eta_{t-2}\right)$ that confines $\eta_{t}$ and $\eta_{t-1}$ with positive probability to an area described by the functions in (3.4). This area can be abstract and depends on the parameters $k$ and $\theta$. In what follows we derive a condition that is easier to verify than Assumption B2 by only focussing on this area close to the origin. Note that if $\eta_{t}$ and $\eta_{t-1}$ given $\eta_{t-2}$ can be arbitrarily small with positive probability, then Assumption B2 is satisfied if the infima are nonzero. To that end we define the function

$$
g(\eta ; \theta):=\sup _{x \in I} \frac{\tilde{u}(x, \eta ; \theta)}{x}
$$

\section{Assumption C.}

C1. For all $\eta \in \mathbb{R}$ and $\theta \in \Theta$ we have $g(\eta ; \theta)<\infty$.

C2. The sequence $\left(\eta_{t}\right)_{t \in \mathbb{Z}}$ is SE. 
C3. There exist a $N \in \mathbb{N}$ such that $\mathbb{P}\left(\left|\eta_{t}\right|<1 / n ;\left|\eta_{t-1}\right|<1 / m \mid \eta_{t-2}\right)>0$ almost surely for all $n, m \geq N$. Also the probability that $\eta_{t}=0$ is zero.

Assumption $\mathrm{C} 1$ is an assumption on the updating function $u$ of model (3.1). The condition is of a similar nature as those found in theory on geometric ergodicity of nonlinear time series, see Cline and $\mathrm{Pu}$ (1999). It implies that the function $\tilde{u}$ as a function of $x$ is bounded on any closed interval, and asymptotically as $x \rightarrow \infty$ is bounded by a linear function. These two facts ensure that the infima in (3.4) are nonzero.

The other conditions are purely on the distribution of $\left(\eta_{t}\right)_{t \in \mathbb{Z}}$. Assumption C3 entails that $\eta_{t}$ and $\eta_{t-1}$ have positive probability of being arbitrarily small, independent of the value of $\eta_{t-2}$. An example on how Assumption C3 can be derived is if $\left(\eta_{t}\right)_{t \in \mathbb{Z}}$ is obtained as a SE solution from another model. For example, suppose that $\left(\eta_{t}\right)_{t \in \mathbb{Z}}$ is given by a SE solution to an autoregressive process of order one

$$
\eta_{t+1}=\beta \eta_{t}+\zeta_{t}
$$

Then a sufficient condition would be that $\left(\zeta_{t}\right)_{t \in \mathbb{Z}}$ is iid, that $\zeta_{t}$ is absolutely continuous with respect to the Lebesque measure on $\mathbb{R}$ and that $\zeta_{t}$ has a strictly positive probability density function. Note that these conditions imply that any set in $B \in \mathcal{B}(\mathbb{R} \times \mathbb{R} \times \mathbb{R})$ has $\mathbb{P}\left(\left(\eta_{t}, \eta_{t-1}, \eta_{t-2}\right) \in B\right)>0$, so in particular Assumption C3 is implied.

If we can assume that the sequence $\left(\eta_{t}\right)_{t \in \mathbb{Z}}$ is independent, then Assumption C simplifies as follows:

\section{Assumption D.}

D1. For all $\eta \in \mathbb{R}$ and $\theta \in \Theta$ we have $g(\eta ; \theta)<\infty$.

D2. The sequence $\left(\eta_{t}\right)_{t \in \mathbb{Z}}$ is iid.

D3. There exist a $N \in \mathbb{N}$ such that $\mathbb{P}\left(\left|\eta_{t}\right|<1 / n\right)>0$ for all $n \geq N$. Also the probability that $\eta_{t}=0$ is zero.

Assumption D3 implies Assumption C3 if $\left(\eta_{t}\right)_{t \in \mathbb{Z}}$ is iid and describes that $\eta_{t}$ being arbitrarily small has positive probability. This, for example, is implied if $\eta_{t}$ is absolutely continuous with respect to the Lebesque measure on $\mathbb{R}$ and the probability density function of $\eta_{t}$ is strictly positive on an open interval around zero. Common distributions such as the normal and student- $t$ distribution satisfy this condition.

Lemma 3.1. Assumption $C$ implies Assumption B.

Proof. We need to check whether Assumption B2 is satisfied. Assumption C1 ensures that the random variable

$$
\inf _{x \in I} \frac{k x \eta_{t-1}^{2}}{\omega+\tilde{u}\left(x, \eta_{t-1}^{2} ; \theta\right)}
$$

is equal to zero if and only if $\eta_{t-1}=0$, since

$$
\inf _{x \in I} \frac{k x \eta_{t-1}^{2}}{\omega+\tilde{u}\left(x, \eta_{t-1}^{2} ; \theta\right)} \geq \inf _{x \in I} \frac{k x \eta_{t-1}^{2}}{\omega+g\left(\eta_{t-1} ; \theta\right) x}=\frac{k \eta_{t-1}^{2}}{1+g\left(\eta_{t-1} ; \theta\right)} .
$$


Assumption C3 therefore implies that $k \eta_{t-1}^{2} /\left(1+g\left(\eta_{t-1} ; \theta\right)\right)$ is nonzero with probability one. Therefore, the probability that

$$
\eta_{t}^{2} \leq \frac{k \eta_{t-1}^{2}}{1+g\left(\eta_{t-1} ; \theta\right)} \quad \text { and } \quad \eta_{t-1}^{2} \leq \frac{k \eta_{t-2}^{2}}{1+g\left(\eta_{t-2} ; \theta\right)}
$$

is greater than zero. This follows, since the infima are nonzero, due to the fact that $\eta_{t}$ and $\eta_{t-1}$ can be arbitrarily small with positive probability so in particular, they have positive probability to be smaller than these upper bounds.

Theorem 3.2. If Assumption $B$ holds, then there exists a solution $\left(\left(\epsilon_{t}, \sigma_{t}^{2}\right)\right)_{t \in \mathbb{Z}}$ to $(3.1)$ given by

$$
\begin{aligned}
\sigma_{t+1}^{2} & =\lim _{m \rightarrow \infty} \phi_{t}^{(m)}(x, y), \\
\epsilon_{t+1} & =\sqrt{\lim _{m \rightarrow \infty} \phi_{t}^{(m)}(x, y)} \eta_{t+1} .
\end{aligned}
$$

This solution is stationary ergodic, unique and any partial solution converges to it at any rate. Moreover, if additionally $\left(\eta_{t}\right)_{t \in \mathbb{Z}}$ is $\varphi$-mixing with geometric rate, then $\left(\left(\epsilon_{t}, \sigma_{t}^{2}\right)\right)_{t \in \mathbb{Z}}$ is $\varphi$-mixing with geometric rate.

Proof. We will start by verifying that assumptions A are all satisfied, so that Theorem 2.1 implies that

$$
\left(\lim _{m \rightarrow \infty} \psi_{t}^{(m)}(x, y)\right)_{t \in \mathbb{Z}}
$$

is a SE and unique solution to (3.3) such that all partial solutions converge to it. Assumption A1 is satisfied by Borel-measurability of $u$. Assumption A2 requires the sequence $\left(\left(\eta_{t}, \eta_{t-1}\right)\right)_{t \in \mathbb{Z}}$ to be SE, which is implied by B1 and Lemma 2.2. Finally, we will show that (3.4) implies that $\psi_{t}^{(3)}(x, y)=(\omega, \omega)$ for all $(x, y) \in S$ and therefore implies Assumption A3. Note that

$$
\phi_{t}^{(2)}(x, y)=\phi_{t}\left(\phi_{t-1}(x, y), x\right)=\omega+\tilde{u}\left(\phi_{t-1}(x, y), \eta_{t} ; \theta\right) \mathbb{1}\left\{\phi_{t-1}(x, y) \eta_{t}^{2}>k x \eta_{t-1}^{2}\right\},
$$

so that $\phi_{t}^{(2)}(x, y)=\omega$ for all $(x, y) \in S$ iff $\eta_{t}^{2} \leq \frac{k x \eta_{t-1}^{2}}{\phi_{t-1}(x, y)}$ for all $(x, y) \in S$, which is implied by

$$
\eta_{t}^{2} \leq \inf _{x \in I} \frac{k x \eta_{t-1}^{2}}{\omega+\tilde{u}\left(x, \eta_{t-1}^{2} ; \theta\right)} .
$$

The first part of the proof is concluded by noting that

$$
\psi_{t}^{(3)}(x, y)=\left(\phi_{t}^{2}\left(\phi_{t-2}(x, y), x\right), \phi_{t-1}^{2}(x, y)\right)=\left(\phi_{t}^{2}(\tilde{x}, \tilde{y}), \phi_{t-1}^{2}(x, y)\right) .
$$

Next, a unique and SE solution to (3.3) to which all partial solutions converge to implies the existence of a solution to (3.2) with the same properties, by projecting on the first coordinate. The found solution is given by

$$
\lim _{m \rightarrow \infty} \phi_{t}^{(m)}(x, y)
$$


which is a measurable function of $\left(\eta_{t-1}, \eta_{t-2}, \ldots\right)$. Therefore $\epsilon_{t}=\sigma_{t} \eta_{t}$ is a measurable function of $\left(\eta_{t}, \eta_{t-1}, \ldots\right)$ and thus (3.5) is a SE solution to (3.1) by Lemma 2.2. Uniqueness and convergence of partial solutions transfer directly from those properties for (3.2).

Finally, suppose $\left(\eta_{t}\right)_{t \in \mathbb{Z}}$ is $\varphi$-mixing with geometric rate. Then $\left(\left(\eta_{t}, \eta_{t-1}\right)\right)_{t \in \mathbb{Z}}$ is $\varphi$ mixing with geometric rate by Lemma 2.5 and thus

$$
\left(\lim _{m \rightarrow \infty} \phi_{t}^{(m)}(x, y), \eta_{t+1}\right)_{t \in \mathbb{Z}}
$$

is $\varphi$-mixing with geometric rate by applying Theorem 2.4 and Lemma 2.5 again. Applying Lemma 2.5 once more shows that $(3.5)$ is $\varphi$-mixing with geometric rate.

\subsection{Examples}

This section discusses a couple of specifications of the updating function $u$ in model (3.1). We assume that the sequence $\left(\eta_{t}\right)_{t \in \mathbb{Z}}$ is $\varphi$-mixing at a geometric rate and satisfies the distributional conditions of either Assumption C or Assumption D. We then display how quickly our theory can be applied by checking whether Assumption C1/D1 holds for these examples.

Example 1 (Saïdi and Zakoian (2006)). First, we consider model (1.1). We repeat it here for readability.

$$
\begin{aligned}
\epsilon_{t} & =\sigma_{t} \eta_{t}, \\
\sigma_{t}^{2} & =\omega+\alpha \epsilon_{t-1}^{2} \mathbb{1}\left\{\epsilon_{t-1}^{2}>k \epsilon_{t-2}^{2}\right\},
\end{aligned}
$$

where $\alpha$ is nonnegative. We have $u\left(\epsilon_{t-1}, \sigma_{t-1}^{2} ; \alpha\right)=\alpha \epsilon_{t-1}^{2}$, which is a measurable and nonnegative function. Moreover, the function $g\left(\eta_{t} ; \alpha\right)=\alpha<\infty$, so Assumption C1 respective D1 is immediately satisfied. Therefore there exists a strictly stationary and $\varphi$-mixing at geometric rate solution to which all partial solutions converge almost surely. Saïdi and Zakoian (2006) assume that $\left(\eta_{t}\right)_{t \in \mathbb{Z}}$ is iid. They then add the assumptions that $\eta_{t}$ is absolutely continuous with respect to the Lebesque measure on $\mathbb{R}$ and $\eta_{t}$ has a strictly positive probability density function. Note that this assumption is stronger than our Assumption D3. Finally, Saïdi and Zakoian (2006) assume that $\mathbb{E} \eta_{t}=0$ and $\mathbb{E} \eta_{t}^{2}=1$, while we don't have any moment conditions at all.

Example 2 (Asymmetric news impact curve). Second, we consider a model that allows the update function to be asymmetric in $\epsilon_{t-1}$ rather than using the quadratic update $\epsilon_{t-1}^{2}$ considered above. In particular, we follow Engle and $\mathrm{Ng}$ (1993) in using the asymmetric news impact curve $u\left(\epsilon_{t-1}, \sigma_{t-1}^{2}\right)=\alpha\left(\epsilon_{t-1}+\delta \sigma_{t-1}\right)^{2}$ and obtain the following model

$$
\begin{aligned}
\epsilon_{t} & =\sigma_{t} \eta_{t}, \\
\sigma_{t}^{2} & =\omega+\alpha\left(\epsilon_{t-1}+\delta \sigma_{t-1}\right)^{2} \mathbb{1}\left\{\epsilon_{t-1}^{2}>k \epsilon_{t-2}^{2}\right\},
\end{aligned}
$$

where $\alpha$ is nonnegative and $\delta \in \mathbb{R}$. Notice how for $\delta<0$, negative returns $\epsilon_{t}$ have greater impact on future volatility $\sigma_{t+1}^{2}$ than positive returns of the same magnitude, thus capturing the empirical regularity known as the leverage effect. In this example we have $\tilde{u}\left(x, \eta_{t} ; \alpha\right)=\alpha x\left(\eta_{t}+\delta\right)^{2}$ and thus $g\left(\eta_{t} ; \alpha\right)=\alpha\left(\eta_{t}+\delta\right)^{2}<\infty$. Therefore, Assumption C1/D1 is satisfied again and thus there exists a strictly stationary and $\varphi$-mixing at geometric rate solution to which all partial solutions converge almost surely. 
Example 3 (Robust volatility update). Finally, we consider a robust nonlinear ARCH model by adopting an update function that is bounded in $\epsilon_{t-1}$ rather than quadratic. In particular, we study a model which embodies the news impact curve of the student- $t$ score volatility model introduced in Creal et al. (2011, 2013) and the beta-t EGARCH model proposed by Harvey (2013),

$$
\begin{aligned}
\epsilon_{t} & =\sigma_{t} \eta_{t}, \quad \eta_{t} \sim t(\lambda) \\
\sigma_{t}^{2} & =\omega+\alpha \frac{\epsilon_{t-1}^{2}}{1+\lambda^{-1} \epsilon_{t-1}^{2}} \mathbb{1}\left\{\epsilon_{t-1}^{2}>k \epsilon_{t-2}^{2}\right\},
\end{aligned}
$$

where $\alpha$ and $\lambda$ are nonnegative. Notice that the innovations $\eta_{t}$ are allowed to be fat tailed. In particular, they belong to the family of student's- $t$ distributed random variables with $\lambda$ degrees of freedom. The updating function of this model becomes more robust (with a lower upper bound) as $\lambda \rightarrow 0$ so that the innovations $\eta_{t}$ become fatter tailed and outliers become more frequent. In contrast, as we approach the Gaussian case by letting $\lambda \rightarrow \infty$, then the updating function reverts back to that of the nonlinear ARCH model considered in Saïdi and Zakoian (2006). We now have $\tilde{u}\left(x, \eta_{t} ; \alpha, \lambda\right)=\alpha \frac{x \eta_{t}^{2}}{1+x \eta_{t}^{2} / \lambda} \leq \alpha \lambda$, thus $g\left(\eta_{t} ; \alpha, \lambda\right) \leq \alpha \lambda / \omega<\infty$ and Assumption C1/D1 is satisfied again. Hence, there exists a strictly stationary and $\varphi$-mixing at geometric rate solution to which all partial solutions converge almost surely.

\section{Moments}

Moment conditions for model (3.1) can be obtained by showing that the moments of the backward iterates have a converging subsequence. To state our result we define

$$
h(\eta ; \theta)=\limsup _{x \rightarrow \infty} \frac{\tilde{u}(x, \eta ; \theta)}{x} .
$$

Theorem 4.1. Let Assumption D hold. Let $p \geq 1$ and $\tilde{\Theta} \subseteq \Theta$ be such that $\mathbb{E}\left|\eta_{t}\right|^{2 p}<\infty$ and $\mathbb{E} g\left(\eta_{t} ; \theta\right)^{p}<\infty$ and

$$
\mathbb{E}\left(h\left(\eta_{t} ; \theta\right) h\left(\eta_{t-1} ; \theta\right) \mathbb{1}\left\{\eta_{t}^{2}>\frac{k \eta_{t-1}^{2}}{h\left(\eta_{t-1} ; \theta\right)}\right\}\right)^{p}<1
$$

for all $\theta \in \tilde{\Theta}$. Then the unique solution to (3.5) has finite absolute $2 p$ 'th moment, that is $\mathbb{E}\left|\epsilon_{t}\right|^{2 p}<\infty$ and $\mathbb{E} \sigma_{t}^{2 p}<\infty$.

Theorem 4.1 is a generalisation of Theorem 3.3 in Saïdi and Zakoian (2006), their assumption to ensure moments in model (1.1) follows as a specific case from our result. The expectation in condition (4.1) can be hard to calculate, because of the indicator function.

Corollary 4.2. Condition (4.1) is implied by

$$
\mathbb{E} h\left(\eta_{t} ; \theta\right)^{p}<1 .
$$

Proof. This follows directly from Assumption D2 and the fact that the indicator function is bounded by one. 
Condition (4.2) is much easier to calculate, but sacrifices flexibility by ignoring the indicator function. Saïdi and Zakoian (2006) show that (4.1) delivers more flexible bounds for model (1.1) than (4.2) when $\eta_{t} \sim \mathrm{N}(0,1)$. We will discuss the examples of Section 3.1 to illustrate how both conditions can be useful.

Proof of Theorem 4.1. By Assumption D2 we have $\mathbb{E}\left|\epsilon_{t}\right|^{2 p}=\mathbb{E}\left|\eta_{t}\right|^{2 p} \mathbb{E} \sigma_{t}^{2 p}$, so we only have to show $\mathbb{E} \sigma_{t}^{2 p}<\infty$. We know by theorem 3.2 that

$$
\sigma_{t}^{2}=\lim _{m \rightarrow \infty} \phi_{t}^{(m)}(x, y)
$$

so by continuity of the norm and Fatou's lemma we have $\mathbb{E} \sigma_{t}^{2 p}<\infty$ if

$$
\liminf _{m \rightarrow \infty} \mathbb{E}\left|\phi_{t}^{(m)}(x, y)\right|^{p}<\infty
$$

We will prove inequality (4.3). To ease notation we will write $\phi_{t}^{m}=\phi_{t}^{(m)}(x, y)$ and suppress the dependence of the functions $g$ and $h$ on $\theta$. We have

$$
\begin{aligned}
\phi_{t}^{m} & =\omega+\tilde{u}\left(\phi_{t-1}^{m-1}, \eta_{t-1}\right) \mathbb{1}\left\{\phi_{t-1}^{m-1} \eta_{t-1}^{2}>k \phi_{t-2}^{m-2} \eta_{t-2}^{2}\right\} \\
& \leq \omega+g\left(\eta_{t-1}\right) \phi_{t-1}^{m-1} \\
& \leq \omega+g\left(\eta_{t-1}\right)\left(\omega+g\left(\eta_{t-2}\right) \phi_{t-2}^{m-2}\right)
\end{aligned}
$$

Let $n \in \mathbb{N}$ be any integer. We separate the problem into three scenarios. Suppose $\phi_{t-1}^{m-1} \leq$ $n$, then $\phi_{t}^{m}$ is bounded by

$$
\omega+g\left(\eta_{t-1}\right) n
$$

If $\phi_{t-2}^{m-2} \leq n$, then $\phi_{t}^{m}$ is bounded by

$$
\omega+g\left(\eta_{t-1}\right)\left(\omega+g\left(\eta_{t-2}\right) n\right)
$$

Finally, suppose $\phi_{t-1}^{m-1}, \phi_{t-2}^{m-2} \geq n$. Define

$$
h_{n}(\eta)=\sup _{x \geq n} \frac{\tilde{u}(x, \eta ; \theta)}{x}
$$

Then, for $n \geq \omega$, we have $h_{n}(\eta) \leq g(\eta)$ and thus

$$
\begin{aligned}
\phi_{t}^{m} & \leq \omega+h_{n}\left(\eta_{t-1}\right) \phi_{t-1}^{m-1} \mathbb{1}\left\{\eta_{t-1}^{2}>\frac{k \phi_{t-2}^{m-2} \eta_{t-2}^{2}}{\omega+\tilde{u}\left(\phi_{t-2}^{m-2}, \eta_{t-2}\right)}\right\} \\
& \leq\left(1+g\left(\eta_{t-1}\right)\right) \omega+h_{n}\left(\eta_{t-1}\right) h_{n}\left(\eta_{t-2}\right) \phi_{t-2}^{m-2} \mathbb{1}\left\{\eta_{t-1}^{2}>\frac{k \eta_{t-2}^{2}}{\omega / n+h_{n}\left(\eta_{t-2}\right)}\right\} .
\end{aligned}
$$

It follows that $\phi_{t}^{m}$ is bounded by the sum of (4.4)-(4.6) and therefore by independence of $\phi_{t-2}^{m-2}$ with $\eta_{t-1}$ and $\eta_{t-2}$ we get by Minkowski's inequality that

$$
\left[\mathbb{E}\left(\phi_{t}^{m}\right)^{p}\right]^{\frac{1}{p}} \leq C(n)+\left[\mathbb{E} f_{n}\left(\eta_{t-1}, \eta_{t-2}\right)^{p}\right]^{\frac{1}{p}}\left[\mathbb{E}\left(\phi_{t-2}^{m-2}\right)^{p}\right]^{\frac{1}{p}}
$$


where $C(n)$ is a finite constant depending on $n$ and

$$
f_{n}\left(\eta_{t-1}, \eta_{t-2}\right)=h_{n}\left(\eta_{t-1}\right) h_{n}\left(\eta_{t-2}\right) \mathbb{1}\left\{\eta_{t-1}^{2}>\frac{k \eta_{t-2}^{2}}{\omega / n+h_{n}\left(\eta_{t-2}\right)}\right\} .
$$

A sufficient condition for (4.3) is to find an appropriate $n \in \mathbb{N}$ such that $\mathbb{E} f_{n}\left(\eta_{t-1}, \eta_{t-2}\right)^{p}<$ 1. This happens for any choice of $n$ that is large enough, as implied by (4.1) and the dominated convergence theorem, because $f_{n}\left(\eta_{t-1}, \eta_{t-2}\right)$ is bounded by $g\left(\eta_{t-1}\right) g\left(\eta_{t-2}\right)$ for large enough $n$ and as $n \rightarrow \infty$ it converges pointwise to

$$
h\left(\eta_{t-1}\right) h\left(\eta_{t-2}\right) \mathbb{1}\left\{\eta_{t-1}^{2}>\frac{k \eta_{t-2}^{2}}{h\left(\eta_{t-2}\right)}\right\} .
$$

Example 1 (Saïdi and Zakoian (2006) continued). Using Theorem 4.1 we can follow the approach of Saïdi and Zakoian (2006) and find the same conditions for model (1.1) that ensure $\mathbb{E}\left|\epsilon_{t}\right|^{2 p}<\infty$ and $\mathbb{E} \sigma_{t}^{2 p}<\infty$. We need $\mu_{2 p}:=\mathbb{E}\left|\eta_{t}\right|^{2 p}<\infty$ and note that it implies $\mathbb{E} g\left(\eta_{t} ; \alpha\right)^{p}=\alpha^{p} \mu_{2 p}<\infty$. In this example condition (4.1) boils down to

$$
\mathbb{E}\left(\alpha^{2} \eta_{t}^{2} \eta_{t-1}^{2} \mathbb{1}\left\{\eta_{t}^{2}>\frac{k}{\alpha}\right\}\right)^{p}<1
$$

Using Hölder's and Markov's inequalities we get for any $m \in \mathbb{N}$ that the expectation in (4.7) is bounded by

$$
\begin{aligned}
\alpha^{2 p} \mu_{2 p} \mathbb{E}\left(\eta_{t}^{2}\left\{\eta_{t}^{2}>\frac{k}{\alpha}\right\}\right)^{p} & \leq \alpha^{2 p} \mu_{2 p} \mu_{2 p m}^{1 / m} \mathbb{P}\left(\eta_{t}^{2 m}>\left(\frac{k}{\alpha}\right)^{m}\right)^{\frac{m-1}{m}} \\
& \leq \alpha^{2 p} \mu_{2 p} \mu_{2 p m}^{1 / m} \mu_{2 m}^{(m-1) / m}\left(\frac{\alpha}{k}\right)^{m-1}
\end{aligned}
$$

Therefore a sufficient condition for (4.1) is

$$
\alpha<\max _{m \in \mathbb{N}}\left(\frac{k^{m-1}}{\mu_{2 p} \mu_{2 p m}^{1 / m} \mu_{2 m}^{(m-1) / m}}\right)^{1 /(2 p+m-1)} .
$$

Example 2 (Asymmetric news impact curve continued). The model with leverage effects requires again $\mu_{2 p}<\infty$, which implies $\mathbb{E} g\left(\eta_{t} ; \alpha\right)^{p}=\alpha^{p} \mathbb{E}\left(\eta_{t}+\delta\right)^{2 p} \leq 2^{2 p-1} \alpha^{p}\left(\mu_{2 p}+\right.$ $\left.|\delta|^{2 p}\right)<\infty$. This model provides an example where the expectation in (4.1) is hard to calculate. The condition here leads to

$$
\mathbb{E}\left(\alpha^{2}\left(\eta_{t}+\delta\right)^{2}\left(\eta_{t-1}+\delta\right)^{2} \mathbb{1}\left\{\eta_{t}^{2}>\frac{k \eta_{t-1}^{2}}{\alpha\left(\eta_{t-1}+\delta\right)^{2}}\right\}\right)^{p}<1,
$$

but we cannot easily use the Markov inequality to bound the indicator function, since this would lead to moments of the reciprocal of $\eta_{t}$. Instead we use (4.2) and get the sufficient condition $\alpha<\left[\mathbb{E}\left(\eta_{t}+\delta\right)^{2 p}\right]^{-1 / p}$ to obtain $\mathbb{E}\left|\epsilon_{t}\right|^{2 p}<\infty$ and $\mathbb{E} \sigma_{t}^{2 p}<\infty$.

Example 3 (Robust volatility update continued). The robust model has a bounded updating function for the volatility, so therefore we immediately know that $\mu_{2 p}<\infty$ is the only condition we need $\mathbb{E}\left|\epsilon_{t}\right|^{2 p}<\infty$ and $\mathbb{E} \sigma_{t}^{2 p}<\infty$. This result also follows from Theorem 4.1 , since $g(\eta ; \alpha, \lambda) \leq \alpha \lambda$ and $h(\eta ; \alpha, \lambda)=0$ for all $\eta \in \mathbb{R}$. 


\section{Conclusion}

This paper has taken the model introduced in Saïdi and Zakoian (2006) as a starting point to introduce a new set of sufficient assumptions that guarantee the existence of a stationary and $\varphi$-mixing solution in a general setting. These assumptions follow from a SRE approach and are generally stated for random functions on separable Banach spaces. The key assumption is Assumption A3, which describes that the random functions have a positive probability to be constant, that is, the outcome is independent of the argument. Note that this can be generalised from being constant to being a random map, as long as the outcome is independent of the argument. Therefore Assumption A3 can be relaxed to: there exists an $M \in \mathbb{N}$ and an event $A \in \mathcal{E}^{M}$ such that $\left(\eta_{t}, \eta_{t-1}, \ldots, \eta_{t-M+1}\right) \in A$ with positive probability and

$$
\left(\eta_{t}, \eta_{t-1}, \ldots, \eta_{t-M+1}\right) \in A \quad \Rightarrow \quad \phi_{t}^{(M)}(x)=\phi_{t}^{(M)}(y) \quad \forall x, y \in S .
$$

We have refrained from doing this, since it was unnecessary for our setting and makes the proofs less readable. Nevertheless, this could be explored in further research.

The main advantages of our results are that they are generally stated and don't require much from the distribution of the underlying process $\left(\eta_{t}\right)_{t \in \mathbb{Z}}$. We illustrate these advantages in Section 3 by introducing a general nonlinear ARCH model that contains model (1.1). We derive sufficient conditions for stationarity and $\varphi$-mixing in two different scenarios. In the first scenario (Assumption C) we assume that the sequence $\left(\eta_{t}\right)_{t \in \mathbb{Z}}$ is $\mathrm{SE}$ and in the second (Assumption D) we assume that $\left(\eta_{t}\right)_{t \in \mathbb{Z}}$ is iid. Saïdi and Zakoian (2006) derive stability conditions for a specific case of model (3.1) and assume from the start that $\left(\eta_{t}\right)_{t \in \mathbb{Z}}$ is iid. In that scenario we have highlighted in Example 1 that our conditions are less strict.

\section{References}

Birkhoff, G. D. (1931). Proof of the ergodic theorem. Proceedings of the National Academy of Sciences, 17(12):656-660.

Bollerslev, T. (1986). Generalized autoregressive conditional heteroskedasticity. Journal of econometrics, 31(3):307-327.

Bougerol, P. (1993). Kalman filtering with random coefficients and contractions. SIAM Journal on Control and Optimization, 31(4):942-959.

Bougerol, P. and Picard, N. (1992a). Stationarity of garch processes and of some nonnegative time series. Journal of econometrics, 52(1-2):115-127.

Bougerol, P. and Picard, N. (1992b). Strict stationarity of generalized autoregressive processes. The Annals of Probability, pages 1714-1730.

Cline, D. B. and Pu, H.-m. H. (1999). Geometric ergodicity of nonlinear time series. Statistica Sinica, pages 1103-1118.

Creal, D., Koopman, S. J., and Lucas, A. (2011). A dynamic multivariate heavy-tailed model for time-varying volatilities and correlations. Journal of Business 85 Economic Statistics, 29(4):552-563. 
Creal, D., Koopman, S. J., and Lucas, A. (2013). Generalized autoregressive score models with applications. Journal of Applied Econometrics, 28(5):777-795.

Diaconis, P. and Freedman, D. (1999). Iterated random functions. SIAM review, 41(1):4576 .

Engle, R. F. (1982). Autoregressive conditional heteroscedasticity with estimates of the variance of united kingdom inflation. Econometrica: Journal of the Econometric Society, pages $987-1007$.

Engle, R. F. and Ng, V. K. (1993). Measuring and testing the impact of news on volatility. The journal of finance, 48(5):1749-1778.

Harvey, A. C. (2013). Dynamic models for volatility and heavy tails: with applications to financial and economic time series, volume 52. Cambridge University Press.

Kallenberg, O. (2002). Foundations of Modern Probability. Probability and Its Applications. Springer New York.

Krengel, U. (1985). Ergodic theorems, volume 6. Walter de Gruyter.

Nelson, D. B. (1991). Conditional heteroskedasticity in asset returns: A new approach. Econometrica: Journal of the Econometric Society, pages 347-370.

Saïdi, Y. (2003). Étude probabiliste et statistique de modèles conditionnellement hétéroscédastiques non linéaires. $\mathrm{PhD}$ thesis, Université de Lille 3.

Saïdi, Y. and Zakoian, J.-M. (2006). Stationarity and geometric ergodicity of a class of nonlinear arch models. The Annals of Applied Probability, 16(4):2256-2271.

Straumann, D. (2005). Estimation in Conditionally Heteroscedastic Time Series Models. Lecture Notes in Statistics, volume 181. Springer, Berlin.

Straumann, D. and Mikosch, T. (2006). Quasi-maximum-likelihood estimation in conditionally heteroscedastic time series: a stochastic recurrence equations approach. The Annals of Statistics, 34(5):2449-2495.

Zakoian, J.-M. (1994). Threshold heteroskedastic models. Journal of Economic Dynamics and control, 18(5):931-955. 